\title{
Relation between Findings of Nerve Conduction Study and Hand Function in Carpal Tunnel Syndrome
}

\author{
NAWAL A. ABU SHADY, Ph.D.*; MYE A. BASHEER, M.D.**; AHMED A. SHEHAB EL-DEIN, M.Sc.* and \\ EMAN YASEN, Ph.D.*** \\ The Departments of Neuromuscular Disorders and its Surgery, Faculty of Physical Therapy* and Special Medicine, \\ Faculty of Medicine**, Cairo University and Physical Therapist College, Human Performance in Health and Disability, \\ Al-Kasr Al-Einy Hospital***
}

\begin{abstract}
Background: Functional problems occur in carpal tunnel syndrome patients affecting their daily living activities.

Aim of Study: The purpose of this study was to investigate the relation between nerve conduction study and hand function findings in carpal tunnel syndrome.

Subjects and Methods: The study included 40 female patients diagnosed with carpal tunnel syndrome. They were assessed by nerve conduction study, pinch dynamometer for pinch strength and Boston questionnaire for assessing the symptoms severity and functional status of patients with CTS

Results: This study revealed that there was no correlation between motor latency and symptoms subscale $(r=0.113, p=$ $0.489)$, functional subscale $(r=0.212, p=0.189)$, total hand function scale $(r=0.177, p=0.274)$, tip pinch $(r=0.093, p=$ $0.567)$, and key pinch $(r=0.025, p=0.88)$ and palmar pinch $(r=0.001, p=0.995)$.
\end{abstract}

There was no correlation between motor amplitude and symptoms subscale $(r=0.018, p=0.911)$, functional subscale $(r=0.075, p=0.645)$, total hand function scale $(r=0.03, p=$ $0.855)$, key pinch $(r=0.196, p=0.225)$ and palmar pinch $(r=$ $0.062, p=0.705)$. While, there was weak positive significant correlation between motor amplitude and tip pinch $(r=0.351$, $\left.p=0.027^{*}\right)$.

There was no correlation between velocity of motor nerve and symptoms subscale $(r=0.199, p=0.217)$, functional subscale $(r=0.245, p=0.127)$, total hand function scale $(r=0.247, p=$ $0.125)$, tip pinch $(r=0.174, p=0.282)$, between, key pinch ( $r=$ $0.049, p=0.764)$, and palmar pinch $(r=0.013, p=0.936)$.

There was no correlation between onset sensory latency and symptoms subscale $(r=0.01, p=0.951)$, functional subscale $(r=0.036, p=0.826)$, total hand function scale $(r=0.013, p=$ $0.935)$, tip pinch $(r=0.095, p=0.56)$, key pinch $(r=0.212$, $p=0.19)$ and palmar pinch $(r=0.188, p=0.245)$.

There was no correlation between sensory amplitude and symptoms subscale ( $r=0.033, p=0.841)$, functional subscale

Correspondence to: Dr. Nawal A. Abu Shady,

The Department of Neuromuscular Disorders and its Surgery, Faculty of Physical Therapy, Cairo University $(r=0.143, p=0.379)$, total hand function scale $(r=0.096, p=$ $0.555)$, tip pinch $(r=0.178, p=0.273)$, key pinch $(r=0.099, p=$ $0.543)$ and palmar pinch $(r=0.019, p=0.909)$.

Conclusion: Hand function assessment should be done separately from nerve conduction study when assessing carpal tunnel syndrome patients. Psychological factors should be considered.

Key Words: Carpal tunnel syndrome - Nerve conduction study - Handfunction findings.

\section{Introduction}

CARPAL Tunnel Syndrome (CTS) is defined as symptomatic compression neuropathy of the median nerve at the level of the wrist [1]. CTS patients presented by hand pain, numbness, and tingling in the distribution of the median nerve (thumb, index, middle finger, and the radial side of the ring finger) and reduction in grip strength and hand function [2]

The thumb is an integral part of complex hand tasks, allowing for the grasping and manipulating of objects, and a slight disturbance in motor function can be disabling. In general the CTS patients had smaller Range of Motion (ROM) [4]. CTS led to a decrease in force accuracy and an increase in amount of force variability, particularly as the force was applied without visual feedback of force production [5].

The relationship between the abnormalities on NCS's to the duration and severity of symptoms and signs of CTS has been studied. Patients with weakness and/or sensory deficits frequently have low amplitude motor and/or sensory potentials, respectively [6]. 
Significant positive correlation was found between median motor distal latency and Boston carpal tunnel syndrome questionnaire for both severity of symptoms and functional status subscales respectively [7].

A statistically significant correlation between electrophysiological severity findings and Boston carpal tunnel syndrome questionnaire, also by increase in Boston carpal tunnel syndrome questionnaire scores, electrophysiological findings were obtained matching with more severe Carpal Tunnel Syndrome (CTS) [8]

Pinch dynamometric test results and motor distal latency is correlated significantly [9]

\section{Subjects and Methods}

This study was conducted to investigate the relation between findings of nerve conduction study and hand function in carpal tunnel syndrome patients. The study took place at Al-Kasr Al-Aini Hospital from Oct. 2016 to Feb. 2017. Forty female patients diagnosed as CTS were selected.

\section{Design of the study:}

Subjects were evaluated by nerve conduction study, hand function questionnaire and pinch dynamometer.

Inclusion criteria: Subjects' age ranged from 30 to 50, patients have pain and parasthesia (numbness, tingling, burning) in the median nerve distribution without extra median nerve territory symptoms, worsening of symptoms at night, self-reported hand strength deficits, positive tinel's sign and positive phalen's sign.

Exclusion criteria: Previous surgery for carpal tunnel syndrome, multiple diagnosis of the upper extremities such as lateral epicondylitis or cervical radiculopathy, previous fracture of the bones of upper extremity, trauma of the neck or ipsilateral shoulder, neurological diseases e.g stroke, brain tumors, thyroid dysfunction, and uncontrolled diabetes mellitus [10].

\section{Procedures:}

The consents have been obtained. The patients were examined according to the neurology sheet for medical history, sensory manifestations and motor manifestations.

\section{Nerve conduction study:}

Median nerve motor study: Recording from Abductor Pollicis Brevis (APB) muscle [11] and stimulating the median nerve at wrist (between the tendons to the flexor carpi radialis and palmaris longus) and antecubital fossa (over the brachial artery pulse) [3]. Antidromic median nerve sensory nerve conduction study: Recording from index finger with G1 placed over the metacarpalphalangeal joint and G2 placed 3-4 cm distally over the distal interphalangeal joint and stimulating the median nerve at wrist( between the tendons to the flexor carpi radialis and palmaris longus) $[4,12]$

Sensory and motor conduction studies of the ulnar nerve were conducted to rule out ulnar nerve involvement e.g. peripheral neuropathy [13]. Ulnar nerve motor study: Recording from Abductor Digiti Minimi (ADM) muscle with G1 placed over the muscle belly and G2 placed over the fifth metacarpal-phalangeal joint and stimulating ulnar nerve at wrist (adjacent to the flexor carpi ulnaris tendon) and below elbow: $3 \mathrm{~cm}$ distal to the medial epicondyle [3]. Antidromic ulnar nerve sensory study: Recording from the little finger with G1placed over the metacarpal-phalangeal joint and G2placed $3-4 \mathrm{~cm}$ distally over the distal interphalangeal joint and stimulating the ulnar nerve at wrist (adjacent to the flexor carpi ulnaris tendon) [12]

Median nerve versus ulnar nerve-lumbricalinterossei studies: Recording from the second lumbrical (median innervated) and first palmar interosseous (ulnar innervated); same recording electrodes for both: G1 : Placed slightly lateral to the midpoint of the third metacarpal and G2: Placed distally over the metacarpal-phalangeal joint of digit 2 [11] and stimulating the median nerve at the wrist (between the tendons to the flexor carpi radialis and palmaris longus) and ulnar nerve at the wrist adjacent to the flexor carpi ulnaris tendon [4]. Median nerve versus ulnar nerve-digit 4 sensory studies: Recording from ring finger (digit 4) with G1: Placed over the metacarpal-phalangeal joint and G2: Placed 3-4cm distally over the distal interphalangeal joint and stimulating median and ulnar nerve at the wrist [13]

Ground electrode placed between stimulator and recording electrodes over a bony prominence to reduce the electrical noise [15]

The patients' nerve conduction study results have been classified according to electrodiagnostic grading scale as early, mild, moderate and severe $[16,17]$

\section{Pinch dynamometer:}

Tip pinch (thumb to index finger), key pinch (thumb pad to lateral aspect of middle phalanx of index) and tripod (palmar) pinch (thumb pad to 
pads of index and middle fingers) [18]. The mean of three trials was calculated and used for analysis. A rest of 10s occurred between trials [13]

\section{Hand function test:}

Boston questionnaire or carpal tunnel questionnaire has been used. It is self-administered and is in two sections: 1) Symptoms Severity Scale (SSS) which composed of eleven questions and it evaluates symptoms regarding severity, frequency, time and kind. 2) Functional Status Scale (FSS) which composed of eight questions and evaluates how the syndrome affects daily life [19]. Responses may be scored one (mildest) point to five (most severe) points [8].

\section{Statistical analysis:}

It was conducted using SPSS for windows, Version 22 (SPSS, Inc., Chicago, IL). Prior to final analysis, data was screened for normality assumption and presence of extreme scores. This exploration was done as a pre-requisite for parametric calculations of the analysis of difference.

Descriptive analysis using histograms with the normal distribution curve showed that the motor and onset sensory latency, motor and sensory amplitude, velocity of motor of median nerve, symptoms subscale, functional subscale, total hand function scale, tip pinch, key pinch, and palmar pinch were normally distributed and not violates the parametric assumption for the measured dependent variable.

Normality test of data using Shapiro-Wilk test was used, that reflect the data was normally distributed for all most dependents variables. Pearson product moment correlation coefficient was used to determine the correlations among the motor and sensory latency, motor and sensory amplitude, velocity of motor of median nerve and symptoms subscale, functional subscale, total hand function scale, tip pinch, key pinch, and palmar pinch. The initial alpha level for the correlation analysis was set at 0.05 .

\section{Results}

As presented at (Table 1) the correlations among nerve conduction study measures and symptoms, subscale, functional subscale, total hand function scale, tip pinch, key pinch, and palmar pinch were studied through the pearson product moment correlation coefficient. It revealed that there was no correlation between motor latency and symptoms subscale ( $r=0.113, p=0.489$ ). As well as, there was no significant correlation between motor latency and functional subscale ( $r=0.212, p=0.189)$. Addi- tionally, there was no correlation between motor latency and total hand function scale ( $r=0.177, p=$ 0.274 ), between motor latency and tip pinch ( $r=$ $-0.093, p=0.567)$, between motor latency and key pinch $(r=-0.025, p=0.88)$, between motor latency and palmar pinch $(r=0.001, p=0.995)$.

There was no correlation between motor amplitude and symptoms subscale ( $r=0.018, p=0.911$ ). While, there was no significant correlation between motor amplitude and functional subscale $(r=-0.075$, $p=0.645$ ). There was no correlation between motor amplitude and total hand function scale ( $r=-0.03$, $p=0.855$ ), while, there was weak positive significant correlation between motor amplitude and tip pinch $(r=0.351, p=0.027 *)$, there was no significant correlation between motor amplitude and key pinch $(r=0.196, p=0.225)$, between motor amplitude and palmar pinch $(r=0.062, p=0.705)$.

There was no correlation between velocity of motor nerve and symptoms subscale $(r=0.199, p=$ 0.217 ). As well as, there was no significant correlation between velocity of motor nerve and functional subscale ( $r=0.245, p=0.127$ ). Additionally, there was no correlation between velocity of motor nerve and total hand function scale ( $r=0.247, p=$ 0.125 ), between velocity of motor nerve and tip pinch ( $r=0.174, p=0.282$ ), between velocity of motor nerve and key pinch ( $r=0.049, p=0.764)$, between velocity of motor nerve and palmar pinch ( $r=-0.013, p=0.936)$.

There was no correlation between onset sensory latency and symptoms subscale $(r=0.01, p=0.951)$. As well as, there was no significant correlation between onset sensory latency and functional subscale ( $r=-0.036, p=0.826$ ). Additionally, there was no correlation between onset sensory latency and total hand function scale ( $r=-0.013, p=0.935$ ), between onset sensory latency and tip pinch ( $r=$ $-0.095, p=0.56$ ), between onset sensory latency and key pinch ( $r=-0.212, p=0.19)$, between onset sensory latency and palmar pinch ( $r=-0.188, p=$ $0.245)$.

There was no correlation between sensory amplitude and symptoms subscale ( $r=0.033, p=0.841$ ) There was no significant correlation between sensory amplitude and functional subscale $(r=0.143$, $p=0.379$ ). Additionally, there was no correlation between sensory amplitude and total hand function scale ( $r=0.096, p=0.555)$, between sensory amplitude and tip pinch ( $r=0.178, p=0.273)$, between sensory amplitude and key pinch $(r=0.099, p=$ 0.543 ), between sensory amplitude and palmar pinch $(r=-0.019, p=0.909)$. 
Table (1): Bivariate correlations among nerve conduction study measures and symptoms, subscale, functional subscale, total hand function scale, tip pinch, key pinch, and palmar pinch.

\begin{tabular}{lcccccc}
\hline & $\begin{array}{c}\text { Symptoms } \\
\text { subscale }\end{array}$ & $\begin{array}{c}\text { Functional } \\
\text { subscale }\end{array}$ & $\begin{array}{c}\text { Total hand } \\
\text { function scale }\end{array}$ & Tip pinch & Key pinch & Palmar pinch \\
\hline Motor latency & $r=0.113$ & $r=0.212$ & $r=0.177$ & $r=-0.093$ & $r=-0.025$ & $r=0.001$ \\
& $p=0.489$ & $p=0.189$ & $p=0.274$ & $p=0.567$ & $p=0.88$ & $p=0.995$ \\
Motor amplitude & $r=0.018$ & $r=-0.075$ & $r=-0.03$ & $r=0.351$ & $r=0.196$ & $r=0.062$ \\
& $p=0.911$ & $p=0.645$ & $p=0.855$ & $p=0.027 *$ & $p=0.225$ & $p=0.705$ \\
Velocity of motor nerve & $r=0.199$ & $r=0.245$ & $r=0.247$ & $r=0.174$ & $r=0.049$ & $r=-0.013$ \\
& $p=0.217$ & $p=0.127$ & $p=0.125$ & $p=0.282$ & $p=0.764$ & $p=0.936$ \\
Onset sensory latency & $r=0.01$ & $r=-0.036$ & $r=-0.013$ & $r=-0.095$ & $r=-0.212$ & $r=-0.188$ \\
& $p=0.951$ & $p=0.826$ & $p=0.935$ & $p=0.56$ & $p=0.19$ & $p=0.245$ \\
Sensory amplitude & $r=0.033$ & $r=0.143$ & $r=0.096$ & $r=0.178$ & $r=0.099$ & $r=-0.019$ \\
& $p=0.841$ & $p=0.379$ & $p=0.555$ & $p=0.273$ & $p=0.543$ & $p=0.909$ \\
\hline *: Significant at alpha level 0.05. & $p$ : Probability value. & $r$ : Pearson correlation. &
\end{tabular}

\section{Discussion}

Because some clinical and physical outcomes in carpal tunnel syndrome patients are potentially modifiable risk factors, a better understanding of the interactions between clinical, physical, and neurophysiological impairments associated with self-reported function in women with CTS may assist clinicians in determining the real problems of CTS patients potentially enhancing the outcomes in this patient population [20].

The results of our study revealed that there was no significant correlation between nerve conduction study findings and hand function findings (Boston questionnaire scores) in carpal tunnel syndrome patients. This is consistent with the results of Leighton et al., 2007 [21] who stated that there were no statistically significant relationships between the electrodiagnostic findings and patient functional status and symptom severity and concluded that electrodiagnostic findings and patient CTS related symptoms and function appear to be independent measures. Clinicians and researchers interested in CTS outcomes need to assess both. They suggest that the impact of patient psychological characteristics has not been thoroughly examined in CTS.

Depression, somatization (tendency to experience and communicate medically unexplained somatic symptoms in response to psychological distress), and tendency to catastrophize (ruminate about pain, appraise pain as highly threatening, and perceive oneself as helpless to control pain) are important variables that have been demonstrated consistently to be associated with pain intensity and functional limitations in a variety of chronic pain patient populations. It is possible that the presence of psychological dysfunction in these areas might override the role of physiologic factors (reflected in electrodiagnostic findings) when assessing CTS symptoms and function [21].

Another study also suggested that motor impairments may be also a perpetuating factor for chronic pain because pain-related fear or avoidance behaviors, which are considered maladaptive processes increasing pain-related disability, may be also involved [22].

César et al. 2013, [20] confirmed that and the result of his study showed that there were significant positive correlations between functional status subscale score and pain, depression severity and duration of symptoms, with higher pain intensity more severe depression and longer symptom duration being associated with higher functional status subscale score (worse function) in women with CTS.

Electrophysiological severity score had a borderline correlation with boston questionnaire scores, while the subjective symptoms of CTS are well correlated with psychological factors. The boston questionnaire scores score correlated well with hospital anxiety and depression scale, suggesting that CTS symptom severity has a direct relation to the psychological status of patients. The electrophysiological severity score was not correlating with psychological status. This means that the symptoms of CTS are correlated more with psychological status of the patient rather than the electrophysiological severity of the disease. The subjective objective discordance in CTS severity is observed [23]

Other study stated that boston questionnaire did not correlate with DSL or DML. There was no 
significant difference in boston questionnaire scores derived probability in patients categorized as having mild, moderate, or severe disease [24].

No significant differences in boston carpal tunnel questionnaire scores among the patients with minimal, moderate, and severe CTS that were found by nerve conduction study, determining a lack of relationship between electrodiagnostic findings and functional deficits [22].

Only modest correlations were found between the values of the different nerve conduction parameters and nights per week waking due to symptoms, main complaint severity, day or night pain, day or night paraesthesia, symptom severity score and functional status score [25].

There was no relationship between the improvements in boston questionnaire scores and the distal conduction in the median nerve post-operative and the degree of improvement in the symptoms and the functional status after release could not be predicted from the pre-surgical boston questionnaire scores [26].

Some manual worker in whom there was no appreciable wasting of the thenar eminence or weakness of thumb abduction, despite electrodiagnostic evidence of bilateral extremely severe median neuropathies. These are clinically important findings, suggesting clinical examination of motor function may not reliably inform us about severity of median nerve pathology. Confounding musculoskeletal pathology is one reason for dissociation between clinical examination and electrical findings [27].

In contrast to our results Noopur et al., 2015, [28] found a statistically significant correlation between Distal Motor Latency (DML), Distal Sensory Latency (DSL) and Sensory Nerve Action Potential amplitude (SNAP) with Boston carpal tunnel syndrome questionnaire (symptom severity scale and functional status scale).

Also a significant positive correlation was found between median motor distal latency and Boston carpal tunnel syndrome questionnaire for both severity of symptoms and functional status subscales respectively [7].

A statistically significant correlation was found between electrophysiological severity findings and Boston carpal tunnel syndrome questionnaire, also by increase in Boston carpal tunnel syndrome questionnaire scores, electrophysiological findings were obtained matching with more severe Carpal Tunnel Syndrome (CTS) [8].

Deficit in pinch grip force is a common feature in carpal tunnel syndrome patients [22]. Our results showed that there isn't a significant correlation between pinch dynamometric measures and nerve conduction study measures, except a weak positive significant correlation was found between the motor amplitude and the tip pinch. Our results agree with results of César et al., 2013, [20] who stated that there were significant negative correlations between functional status subscale score and pinch grip force of the index finger with lower pinch grip force being associated with a higher functional status subscale score (worse function). Also there were lower pinch grip force compared with the healthy, but there were no differences among those with minimal, moderate, or severe CTS that were classified according to nerve conduction study.

There a study showed that there is no significant associations between pinch grip force and median nerve distal sensory latency or distal motor latency in CTS patients [22]

In contrast to our results Marwa 2010, [9] found that pinch dynamometric test and motor distal latency test are a correlated significantly.

Determining the mechanisms involved in bilateral decrease in pinch grip force in even in unilateral CTS is questionable. Few hypotheses can be proposed. It has been suggested that central mechanisms can be involved. This could lead to impairments in pinch grip force and fine motor control skills in the contralateral extremity, due to a segmental sensitization. Another hypothesis was that bilateral motor control impairment and pinch grip force deficit can reflect a reorganization of the motor control strategy of the central nervous system as a consequence of the pain [13].

Some understanding regarding the pathophysiology of compression neuropathies has come from animal studies. The typical classification of nerve injury (neurapraxia, axonotmesis and neurotmesis) is inadequate to describe the majority of pathophysiologic changes seen in CTS. The lack of human studies on this topic requires us to rely on animal studies. Unfortunately, there are no animal models that accurately and completely simulate CTS [29]

Complete understanding of pathophysiology is needed to figure out the problems of the CTS patients especially the functional problems. 


\section{References}

1- AROORI S. and ROY A.J.: Review Carpal Tunnel Syndrome. Ulster Medical Journal, 77 (1): p. 7, 2008.

2- BURTON C., DAVENPORT G. and LINDA S.: Diagnosing and Managing Carpal Tunnel Syndrome in Primary Care. British Journal of General Practice, 64: p. 262, 2014.

3- LYELL K.: Nerve Conduction Studies: Basic Concepts and Patterns of Abnormalities. Neurologic Clinics Journal, 30 (2): p. 409, 2012.

4- JAMES C.: The Electrodiagnostic Approach to Carpal Tunnel Syndrome. Neurologic Clinics Journal, 30 (2): pp. 460-5, 2012.

5- ZONG-MING L., PETER J., WILLIAM H. and LI K.: Carpal Tunnel Syndrome Impairs Sustained Precision Pinch Performance. Journal of Clinical Neurophysiology, 126 (1): p. 2, 2015.

6- MICHAEL T., YUEN T., DENNIS E. and FAREN H.: Literature Review of the Usefulness of Nerve Conduction Studies and Electromyography For the Evaluation of Patients with Carpal Tunnel Syndrome. Muscle \& Nerve, 16: p. 1404, 1993.

7- CELIK B., PAKER N., COSKUN E., SOY D. and KADRIYE O.: Correlation between median motor studies and duruoz hand index and boston carpal tunnel syndrome questionnaire in patients with carpal tunnel syndrome, in: 18th European Congress of Physical \& Rehabilitation Medicine [poster], Thessaloniki, Greece, 28 th May-1 st June, 2012.

8- ILHAN D., TOKER S., KILINCIOGLU V. and GÜLCAN E.: Assessment of the Boston Questionnaire in Diagnosis of Idiopathic Carpal Tunnel Syndrome: Comparing Scores with Clinical and Neurophysiological Findings. Journal of Duzce Medical School, 3: pp. 4-5, 2008.

9- MOHAMMAD M.: Utilization of wrist caliper and pinch dynamometer in assessment of Carpal tunnel syndrome, MSc Physical Therapy Department for Neuromuscular and Neurosurgical Disorder and its Surgery, Cairo University, 2010.

10- ERKOL E. and EROGLU P.: Analysis of the Relationships between Electroneurography and Boston Questionnaire in Obese Female Patients with Carpal Tunnel Syndrome. Journal of Health Science Institute, 6 (2): p. 73, 2015.

11- ROBERT A. and ANDARY M.: AANEM Monograph Electrodiagnostic Evaluation of Carpal Tunnel Syndrome. Muscle \& Nerve Journal, 44, pp. 597-602, 2011.

12- YILMAZA O., SUNTERA G., SALCINIA C., KAHRAMAN P., TANRIDAGA T., USA O. and ULUCA K.: Motor-unit Number Estimation Is Sensitive in Detecting Motor Nerve Involvement in Patients with Carpal Tunnel Syndrome. Journal of Clinical Neurology, 12 (2): pp. 1667, 2016.

13- FERNÁNDEZ C., PÉREZ M., MARTÍNEZ-PIÉDROLA R., ISABEL A. and JOSHUA A.: Bilateral deficits in fine motor control and pinch grip force in patients with unilateral carpal tunnel syndrome. Experimental Brain Research Journal, 194: pp. 30-5, 2009.

14- EMAN A., ABEER K. and NOURAN M.: The Second Lumbrical-interossei Latency Difference in Carpal Tunnel
Syndrome: Is It a Mandatory Or a Dispensable Test? Alexandria Journal of Medicine, 49: pp. 200-2, 2013.

15- PRESTON D.C. and SHAPIRO B.E.: Electromyography and Neuromusçuarar Disorders: Clinical-electrophysiologic Correlations. ${ }^{\text {3rd }}$ ed. London: Elsevier Saunders, 2013.

16- PADUA L., TONALI P., PADUA R., VALENTE E.M., GREGORI B. and LOMONACO M.: Neurophysiological classification and sensitivity in 500 carpal tunnel syndrome hands. Acta Neurologica Scandinavica Journal, 96: p. 213, 1997.

17- CLARKE J.: AAEM Minimonograph \# 26: The electrodiagnosis of carpal tunnel syndrome. Muscle \& Nerve Journal, 20: p. 1483, 1997.

18- HAN S.H., NAM K.S., CHO Y.S. and RYU K.J.: Normative Data on Hand Grip Strength. Novel Physiotherapies Journal, 1 (1): pp 1-3, 2011.

19- FISCHER J., NEVILLE W. and JOHN W.K.: A Selfadministered Questionnaire for the Assessment of Severity of Symptoms and Functional Status in Carpal Tunnel Syndrome. The Journal of Bone and Joint Surgery Amer ican Volume, 75: p. 394, 1993.

20- FERNÁNDEZ C., JOSHUA A., PLAZA-MANZANO G., ORTEGA-SANTIAGO R., ANA I., MARTÍNEZ-PEREZ A. and ARROYO-MORALES M.: Clinical, Physical, and Neurophysiological Impairments Associated with Decreased Function in Women with Carpal Tunnel Syndrome. Journal of Orthopaedic \& Sports Physical Therapy, 43 (9): pp. 644-6, 2013.

21- CHAN L., TURNER J.A., COMSTOCK B.A., LEVENSON L.M., HOLLINGWORTH W., HEAGERTY P.J., KLIOT M. and JARVIK J.G.: The Relationship Between Electrodiagnostic Findings and Patient Symptoms and Function in Carpal Tunnel Syndrome. Archives of Physical Medicine and Rehabilitation, 88: p. 19, 2007.

22- ISABEL A., FERNÁNDEZ C., PÉREZ M., MART1ḰEZPEREZ A., CARMEN M. and JUAN A.: Bilateral Deficits in Fine Motor Control and Pinch Grip Force Are Not Associated with Electrodiagnostic Findings in Women with Carpal Tunnel Syndrome. American Journal of Physical Medicine \& Rehabilitation, 90: pp. 447-50, 2011.

23- KHAN F., SHEHNA A., RAMESH S., SANDHYA K.S. and PAUL R.: Subjective symptoms of carpal tunnel syndrome correlate more with psychological factors than electrophysiological severity. Annals of Indian Academy of Neurology, 20: pp. 69-72, 2017.

24- MAKANJI H.S., ZHAO M., MUDGAL C.S., JUPITER J.B. and RING D.: Correspondence Between Clinical Presentation and Electrophysiological Testing For Potential Carpal Tunnel Syndrome. Journal of Hand Surgery, 38 (5): p. 492, 2012.

25- HANS M., ANNETTE A.M., ROB L.M., BERNARD M.J., ROB J.P.M., HENRICA C.W. and LEX M.: Journal of Clinical Neurophysiology. Correlating Nerve Conduction Studies and Clinical Outcome Measures on Carpal Tunnel Syndrome: Lessons From a Randomized Controlled Trial, 22 (3), p. 218, 2005.

26- MONDELLI M., REALE F., SICURELLI F. and PADUA L.: Relationship Between the Self-administered Boston Questionnaire and Electrophysiological Findings in Fol- 
low-up of Surgically-treated Carpal Tunnel Syndrome. Journal of Hand Surgery, 25B (2): p. 128, 2000.

27- MARTINS S., CARVILL N. and TAYLOR J.: Clinical Examination Versus Electrodiagnostic Assessment of Severity of Median Nerve Pathology in Carpal Tunnel Syndrome. Journal of Clinical Rheumatology, 22 (1): p. 46, 2016.
28- NOOPUR G., MEGHA S. and NEETA J.: Relationship of Electrodiagnostic Findings with Severity of Symptoms and Function in Subjects with Carpal Tunnel Syndrome. International Journal of Therapies and Rehabilitation Research, 4 (3): p. 1, 2015.

29- ROBERT A. and ANDARY M.: Carpal Tunnel Syndrome: Pathophysiology and Clinical Neurophysiology. Clinical Neurophysiology Journal, 113: p. 1381, 2000.

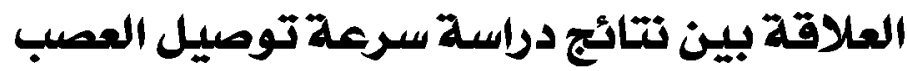

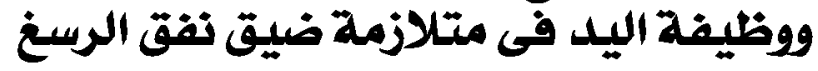

هدذ البحث: التحقق ما إذا كان هناك علاقة بين نتائج دراسة سرعة توصيل العصب ووظيفة اليد في متلازمة ضيق نفق الرسغ.

تم إختيار • ع مريضة يعانون من متلازمة ضيق نفق الربسن وقد تم تقييم قوة القرص عن طريق آداة المقوى وتم تقيبم حدة الآعراض والحالة

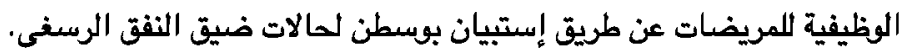

آظهرت النتائج عدم وجود علاقة ذات دلالة إحصائية واضحة بين نتائج وظيفة اليد ونتائج دراسة سرعة توصيل العصب في متلازمة ضيق نفق الرسنغ.

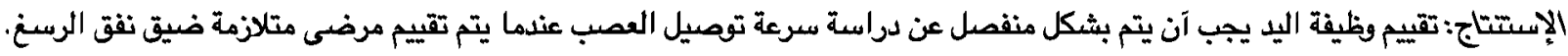
وينبفى النظر فى العوامل النفسية المريض. 